\title{
The Elastic Constants of the Single Crystal of the Mg-Zn-Zr-REM Alloy from the Data of the Elastic Anisotropy and the Texture of the Polycrystalline Sheet
}

\author{
S. V. San'kova, N. M. Shkatulyak, V. V. Usov, and N. A. Volchok \\ South Ukrainian National Pedagogical University, 26 Staroportofrankovskaya Street, Odessa 65020, Ukraine \\ Correspondence should be addressed to V. V. Usov; valentin_usov50@mail.ru
}

Received 31 May 2014; Revised 6 October 2014; Accepted 28 October 2014; Published 13 November 2014

Academic Editor: Manoj Gupta

Copyright (C) 2014 S. V. San'kova et al. This is an open access article distributed under the Creative Commons Attribution License, which permits unrestricted use, distribution, and reproduction in any medium, provided the original work is properly cited.

\begin{abstract}
The measuring of the constants of single-crystals requires the availability of crystals of relatively big size. In this paper the elastic constants of the single crystals of magnesium alloy with zinc, zirconium, and rare earth metals (REM) were determined by means of the experimental anisotropy of Young's modulus and integral characteristics of texture (ICT), which were found from pole figures. Using these constants the anisotropy of Young's modulus of alloy sheet ZE10 was calculated. Deviation of calculated values from experimental values did not exceed $2 \%$.
\end{abstract}

\section{Introduction}

Magnesium alloys attracted attention in recent years because they are the lightest among available metallic commercial materials. Due to the low density $\left(1740 \mathrm{~kg} / \mathrm{m}^{3}\right)$ and a relatively high specific strength, magnesium alloys enable the reduction of weight of construction by replacing the steel and aluminum parts in the aerospace and the transport industry. High resistance to the formability due to unfavorable texture, which was formed in the process of its production, impedes wide use of magnesium alloys in practice. Magnesium alloys with zinc, modified by rare earth metals (REM), such as cerium, neodymium, yttrium, were created with the aim to weaken the undesirable texture by different thermomechanical treatments [1]. However, many questions of formation of texture and anisotropy of properties (in particular, elastic) at different types of heat treatment and deformation of magnesium alloys with zinc, zirconium, and rare earth metals have not yet been sufficiently studied.

Elastic modules and constants of compliances of single crystals are included in all equations of physics and mechanics of deformable solids. Elastic constants are determined of appropriate experiments on single crystals. Growing the artificial single crystals of the desired size is associated with technical difficulties. Using the elastic constants of a single crystal of pure metal for calculating properties of alloys in polycrystalline state can lead to significant errors.

The aim of this work is to determine the elastic constants of a single crystal of magnesium alloy with zinc, zirconium, and rare earth metals (REM) from the data of the measurements of the anisotropy of Young's modulus and integral characteristics of texture (ICT).

\section{Materials and Methods}

Magnesium alloy ZE10 containing $1.3 \% \mathrm{Zn}, 0.15 \% \mathrm{Zr}$, and $0.2 \%$ of REM (mainly cerium) of $1 \mathrm{~mm}$ thickness was used as material for studies. Before the study the alloy was treated according to industrial technology [2]. From these sheets of alloy we cut out seven rectangular specimens of size $100 \times$ $10 \mathrm{~mm}$ through every $15^{\circ}$ from the rolling direction (RD) up to the transverse direction (TD) of sheet for measuring of anisotropy of Young's modulus. Young's modulus was measured by a dynamic method on the frequency of flexural oscillations of a flat sample [3]. Samples were processed in a package to reduce measurement errors due to geometrical dimensions. The error did not exceed $1 \%$. 
The elastic anisotropy of policrystal is determined by the properties of single crystal and crystallographic texture of the policrystal. The grain structure of metal may also play a certain role there. If the relationship between elastic properties of polycrystalline material and its texture is known then it is possible to find the elastic constants of the single crystal or a combination of them. The orientation of a crystal relatively the system of coordinates of the sample is given by means of three variables. The most complete description of the texture, taking into account all its details, is given by the three-dimensional orientation distribution function (ODF) of the crystals. Experimental pole figures (PF) contain the data for calculation of ODF in nonobvious kind. Pole figures are functions of two variables because the PF are the result of the convolutions of three-dimensional ODF [4]. Information about texture in ODF is redundant for calculation of anisotropy of properties in the approximation of continuum mechanics [4]. Therefore for the estimation of the anisotropy of the properties on the texture analysis data it is sufficient to use not the complete ODF but some characteristics of ODF, which contain sufficient information about the anisotropy of the properties. Such characteristics are the certain combinations of directional cosines, averaged according to the law of distribution of crystals on orientations, and named integral characteristics of texture (ICT) $[5,6]$ :

$$
\begin{array}{lll}
I_{1}=\left\langle\alpha_{13}^{2}\right\rangle ; & I_{2}=\left\langle\alpha_{23}^{2}\right\rangle ; & I_{3}=\left\langle\alpha_{33}^{2}\right\rangle ; \\
I_{4}=\left\langle\alpha_{13}^{4}\right\rangle ; & I_{5}=\left\langle\alpha_{33}^{4}\right\rangle ; & I_{6}=\left\langle\alpha_{13}^{2} \cdot \alpha_{23}^{2}\right\rangle .
\end{array}
$$

Here $\alpha_{i k}$ are cosines of angles that set the orientation of the crystal relative to the system of coordinates of the sample; the brackets denote the averaging on all possible orientations of the crystals. The ICT of the orthorhombic polycrystalline objects with a hexagonal lattice may be found $[5,6]$ by averaging of combinations of the direction cosines of the axis $C$ of hexagonal crystal relative to the coordinate system of sample. The coordinate axes of this system are the rolling direction (RD), the transverse direction (TD), and the normal direction (ND) to the sheet plane. Only five ICT are independent for textures of sheets of hexagonal polycrystalline materials, because $I_{1}+I_{2}+I_{3}=1$. Averaging can be performed using direct PF of isotropic plane of the crystal. For hexagonal metal this may be PF $\{0002\}$. Then (1) takes the form $[5,6]$ :

$$
\begin{aligned}
& I_{1}=\frac{1}{2 \pi} \int_{0}^{\pi / 2} \int_{0}^{2 \pi} \sin ^{3} \alpha \cos ^{2} \beta P_{(0002)}(\alpha, \beta) d \alpha d \beta \\
& I_{2}=\frac{1}{2 \pi} \int_{0}^{\pi / 2} \int_{0}^{2 \pi} \sin ^{3} \alpha \sin ^{2} \beta P_{(0002)}(\alpha, \beta) d \alpha d \beta \\
& I_{3}=\frac{1}{2 \pi} \int_{0}^{\pi / 2} \int_{0}^{2 \pi} \sin \alpha \cos ^{2} \beta P_{(0002)}(\alpha, \beta) d \alpha d \beta \\
& I_{4}=\frac{1}{2 \pi} \int_{0}^{\pi / 2} \int_{0}^{2 \pi} \sin ^{5} \alpha \cos ^{4} \beta P_{(0002)}(\alpha, \beta) d \alpha d \beta \\
& I_{5}=\frac{1}{2 \pi} \int_{0}^{\pi / 2} \int_{0}^{2 \pi} \sin ^{5} \alpha \sin ^{4} \beta P_{(0002)}(\alpha, \beta) d \alpha d \beta \\
& I_{6}=\frac{1}{2 \pi} \int_{0}^{\pi / 2} \int_{0}^{2 \pi} \sin ^{5} \alpha \cos ^{2} \beta P_{(0002)}(\alpha, \beta) d \alpha d \beta .
\end{aligned}
$$

Here $P_{(0002)}(\alpha, \beta)$ is the pole density in the corresponding points of $\mathrm{PF}\{0002\} ; \alpha, \beta$ are azimuth and meridian angles of the exit of the pole [0002] on the projection sphere. Anisotropy of Young's modulus in the plane of sheet takes the form [7]:

$$
E(\varphi)=\left[s_{11}+2 a \psi_{2}^{T}(\varphi)+b \psi_{4}^{T}\right]^{-1}
$$

Here

$$
\begin{gathered}
\psi_{2}^{T}(\varphi)=I_{1}^{(h)} \cos ^{4} \varphi+I_{2}^{(h)} \sin ^{4} \varphi+\frac{1}{4}\left(I_{1}^{(h)}+I_{2}^{(h)}\right) \sin ^{2} 2 \varphi \\
\psi_{4}^{T}=I_{6}^{(h)} \cos ^{4} \varphi+I_{4}^{(h)} \sin ^{4} \varphi+1.5 I_{5}^{(h)} \sin ^{2} 2 \varphi \\
a=s_{13}-s_{11}+\frac{1}{2} s_{44} \\
b=s_{11}+s_{33}-2 s_{13}-s_{44} .
\end{gathered}
$$

Young's modulus in the ND will be found according to the formula

$$
\begin{aligned}
\left(E_{H H}\right)^{-1}= & s_{11}+a I_{3}^{(h)} \\
& +b\left(1-2 I_{1}^{(h)}-2 I_{2}^{(h)}+I_{4}^{(h)}+I_{5}^{(h)}+2 I_{6}^{(h)}\right) .
\end{aligned}
$$

Anisotropy of shear modulus in the plane of quasisingle crystal of orthorhombic symmetry (sheet) is expressed as

$$
G(\varphi)=\left[c+d \psi_{2}^{T}(\varphi)+2 b \psi_{4}^{T}\right]^{-1} .
$$

Here $b$ is defined by (7), and $c$ and $d$ are expressed as

$$
\begin{gathered}
c=\frac{1}{2} s_{44}+s_{11}-s_{12}, \\
d=s_{11}-2 s_{33}-4 s_{13}-\frac{3}{2} s_{44}+s_{12} .
\end{gathered}
$$

The functions $\psi_{2}^{T}(\varphi)$ and $\psi_{4}^{T}(\varphi)$ do not depend on properties of the individual crystals. They define only the texture of sheets. Together with single-crystal characteristics they are present in the expression for the anisotropy of properties that are accessible to the tensor description. These functions describe distribution of certain texture characteristics depending on the direction in the plane of sheet or in directions, which form some angles with the plane of sheet. In fact they describe the anisotropy of the orthorhombic sample with hexagonal structure of its inner-element. The anisotropy of the elastic properties in the textured sheet can be represented by a Fourier series with the even harmonics with sufficient accuracy as [7]

$$
E^{-1}(\varphi)=A_{0}+A_{2} \cos 2 \varphi+A_{4} \cos 4 \varphi .
$$

Amplitudes of harmonics (Fourier coefficients) of the series (11) in terms of ICT and elastic constants for hexagonal metals are expressed as [8]

$$
\begin{aligned}
& A_{0}=s_{11}+a\left(I_{1}+I_{2}\right)+\frac{3}{8} b\left(I_{4}+2 I_{5}+I_{6}\right), \\
& A_{2}=\frac{1}{2}\left[a\left(I_{1}-I_{2}\right)+b\left(I_{6}-I_{4}\right)\right], \\
& A_{4}=\frac{1}{8} b\left(I_{4}+I_{6}-6 I_{5}\right),
\end{aligned}
$$




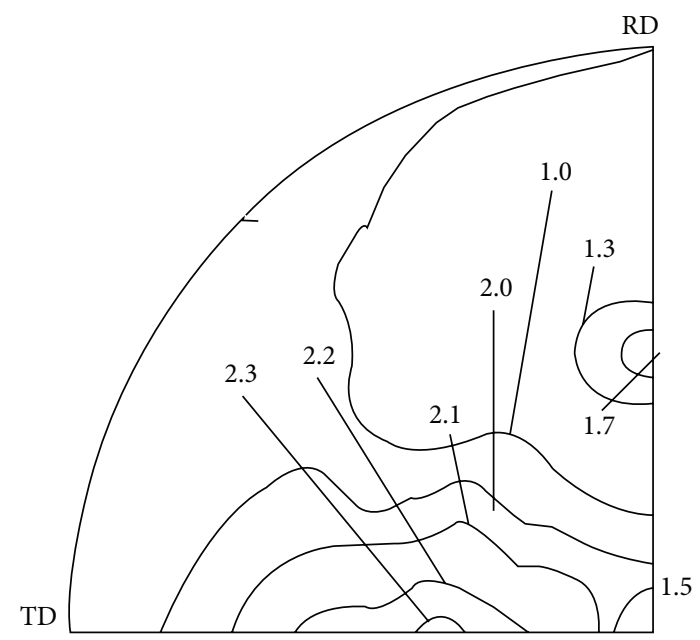

FIgURE 1: Pole figure $\{0002\}$ for the sheet of the alloy ZE10.

If experimental anisotropy of $1 / E$ is represented as a Fourier series and is defined experimentally, then we can find constants $s_{11}$ of alloy and some combinations of them:

$$
\begin{aligned}
s_{11}= & A_{0}-\frac{A_{2}\left(I_{1}+I_{2}\right)}{I_{1}-I_{2}}+4 \frac{A_{4}\left(I_{6}-I_{4}\right)\left(I_{1}+I_{2}\right)}{\left(I_{1}-I_{2}\right)\left(I_{4}+I_{6}-6 I_{5}\right)} \\
& -3 \frac{A_{4}\left(I_{4}+I_{6}+2 I_{5}\right)}{\left(I_{4}+I_{6}-6 I_{5}\right)}, \\
a= & \frac{2 A_{2}}{I_{1}-I_{2}}-8 A_{4} \frac{I_{6}-I_{4}}{\left(I_{1}-I_{2}\right)\left(I_{4}+I_{6}-6 I_{5}\right)}, \\
b= & \frac{8 A_{4}}{\left(I_{4}+I_{6}+6 I_{5}\right)} .
\end{aligned}
$$

Similar data on the anisotropy of a shear modulus will allow to write three equations which relate the characteristics of the single crystal with the amplitudes of the harmonics Fourier series via the ICT and to find all the constants of compliances.

Above mentioned ICT were calculated from (2) using data of the pole figure $\{0002\}$. X-ray technique was used for constructing this PF [9]. Pole density in a particular point of the PF was found from the pole density curves normalized by means of standard sample without the texture (powder sample). Such sample has been prepared from recrystallized saw dusts ZE10 alloy.

\section{Results and Discussions}

The PF $\{0002\}$ for the sheet of the alloy ZE10 is presented in Figure 1.

The texture is characterized by two maxima of pole density at the angular distances from $\mathrm{RD}$ and TD in $45^{\circ}$. The texture of alloy ZE10 sheets differs considerably from the texture of central base type of pure magnesium [1].

Table 1 shows the ICT calculated from (2) and PF data (Figure 1).
TABLE 1: Integral characteristics of texture of alloy ZE10.

\begin{tabular}{lccccc}
\hline \multicolumn{5}{c}{ ICT } \\
\\
$I_{1}$ & $I_{2}$ & $I_{3}$ & $I_{4}$ & $I_{5}$ & $I_{6}$ \\
\hline 0.267466 & 0.327686 & 0.404848 & 0.150986 & 0.191116 & 0.04711 \\
\hline
\end{tabular}

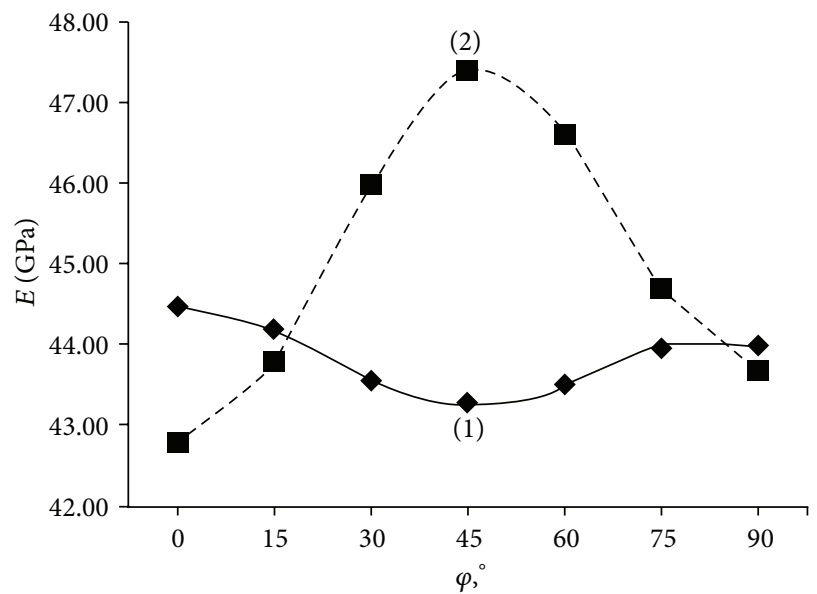

FIGURE 2: Anisotropy of Young's modulus in the sheet of the alloy ZE10. (1) Experiment; (2) calculation by elastic constants of $\mathrm{Mg}$ single crystal.

Table 2 shows the elastic constants of a single crystal of magnesium, taken from [10].

Table 3 shows the experimental anisotropy of Young's modulus that was calculated according to the relation (11). It is easy to see that the anisotropy of Young's modulus expressed as a Fourier series (harmonic model) with 4 even harmonics describes the experimental anisotropy of alloy ZE10 with a deviation not more than $1 \%$. We have calculated the anisotropy of Young's modulus in the alloy ZE10 sheet, using ICT (Table 1) and the elastic constants of a single crystal of pure magnesium (Table 2).

The comparison of the theoretical anisotropy with corresponding experimental data showed satisfactory consent. Results are shown in Figure 2.

Figure 2 shows that the calculated curve does not coincide to the experimental data not only quantitatively but also qualitatively. The experimental curve exhibits a minimum at $45^{\circ}$ to the $\mathrm{RD}$, while the calculated curve shows the maximum here. Thus, the use of the elastic constants of single crystal $\mathrm{Mg}$ in calculation of Young's modulus anisotropy in the alloy ZE10 leads to significant errors. We have calculated the constant of compliances $s_{11}$ and combinations of $a, b$ for the alloy ZE10 by formulas (12) using Fourier coefficients found earlier (Table 3) and ICT (Table 1). We obtained the following values:

$$
\left(s_{11}=2.287 ; a=-0.100 ; b=0.128\right) \cdot 10^{-11} \mathrm{~Pa}^{-1} .
$$

Values of $a, b$ were defined by means (6) and (7).

Next we used the values of the elastic constants obtained from (14) and ICT (Table 1) for estimation of Young's modulus anisotropy in the alloy ZE10 sheet using relationship reverse 
TABLE 2: The elastic constants and some of its combinations for Mg single crystal [10].

\begin{tabular}{lccccccc}
\hline \multirow{2}{*}{ Single crystal } & \multicolumn{4}{c}{ The elastic constants $S_{i j} \cdot 10^{-11}, \mathrm{~Pa}^{-1}$} & \multicolumn{2}{c}{ Combinations $(a, b) \cdot 10^{-11}, \mathrm{~Pa}^{-1}$ according to $(6)$ and $(7)$} \\
& $S_{11}$ & $S_{12}$ & $S_{44}$ & $S_{33}$ & $S_{13}$ & $a$ & $b$ \\
\hline Magnesium & 2.213 & -0.771 & 6.024 & 1.975 & -0.491 & 0.308 & -0.854 \\
\hline
\end{tabular}

TABLE 3: Experimental and Fourier model of anisotropy of Young's modulus in sheet of alloy ZE10.

\begin{tabular}{|c|c|c|c|c|c|c|}
\hline \multirow{2}{*}{$\varphi,^{\circ}$} & \multicolumn{2}{|c|}{$E^{-1} \cdot 10^{-11}, \mathrm{~Pa}^{-1}$} & \multirow{2}{*}{$\left(\Delta E^{-1}\right) / E^{-1}, \%$} & \multicolumn{3}{|c|}{ Fourier coefficients, $\mathrm{Pa}^{-1}$} \\
\hline & Experiment & Model & & $A_{0} \cdot 10^{-11}$ & $A_{2} \cdot 10^{-14}$ & $A_{4} \cdot 10^{-13}$ \\
\hline 0 & 2.25 & 2.27 & -0.89 & & & \\
\hline 15 & 2.26 & 2.27 & -0.44 & & & \\
\hline 30 & 2.30 & 2.29 & 0.43 & & & \\
\hline 45 & 2.31 & 2.30 & 0.43 & 2.29 & -3.64 & -1.52 \\
\hline 60 & 2.30 & 2.29 & 0.43 & & & \\
\hline 75 & 2.27 & 2.28 & -0.44 & & & \\
\hline 90 & 2.27 & 2.27 & 0.00 & & & \\
\hline
\end{tabular}

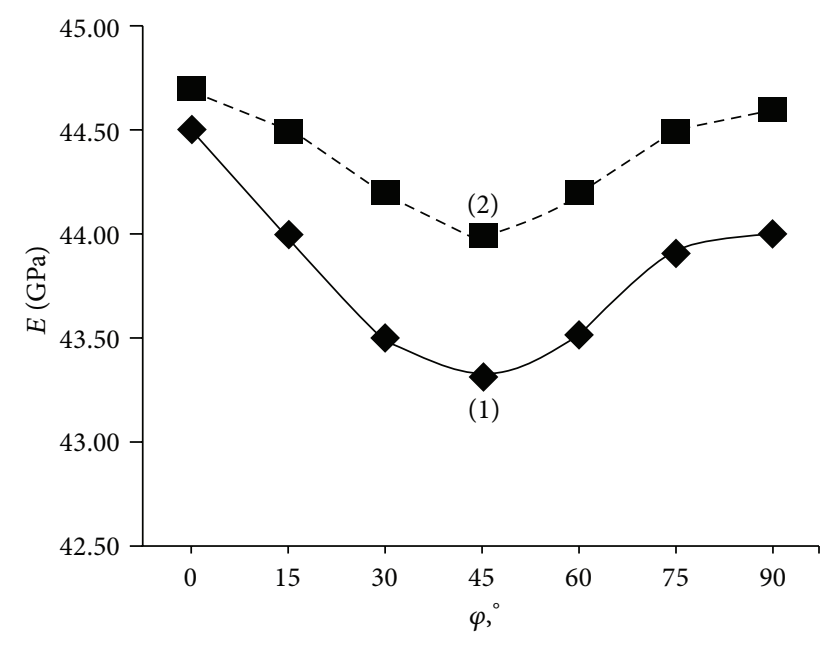

FIGURE 3: Anisotropy of Young's modulus in the sheet of the alloy ZE10. (1) Experiment; (2) calculation by elastic constants of alloy ZE10 single crystal.

to (3) and compared with experiment. The results are shown on Figure 3. Analysis showed that the deviation of the calculated and experimental data does not exceed $2 \%$.

Additionally we have calculated also the value of Young's modulus in ND (END) of the sheet using the relationship reverse to (8), ICT (Table 1), and combination of constants of single crystal (14) of alloy ZE10. We obtained that END = $43.84 \mathrm{GPa}$.

In principle, even if the number of grains is large enough, Young's modulus depends on the orientation of each grain of the textured material.

In order to estimate Young's modulus of such structures, it is important to identify the local properties of different grains relatively to their orientation. Once the crystal orientation of each grain or its statistical distribution is identified, Young's modulus of the aggregate can be evaluated by averaging Young's modulus of each grain based on a geometrical assumption. However, the geometrical condition (uniform local strain or stress) in a polycrystal is not obvious due to the complexity of the geometrical structure of a crystal grain. Furthermore, the complex geometry causes nonuniform stresses at the microstructural level even under a uniform remote stress condition. Deformation of one grain becomes difficult due the presence of neighboring grains. It leads to additional stresses. The variation of Young's modulus from one grain to another as well induces the large stress (or strain) near the grain boundary. Such nonuniform stress may affect the macroscopic Young's modulus [11]. In order to quantify the effect of complex geometry and the local stress distribution in the polycrystalline material, it is necessary to use a numerical approach such as the finite element method [11]. Numerical simulations have shown that the crystal orientation makes the main contribution to the value of Young's modulus, when the number of grains is large enough. The error due to ignoring of the structure is the average of less than $0.3 \mathrm{GPa}$ [11]. For Young's modulus of alloy ZE10 investigated by us this error amounted to $0.7 \%$. This is included in above mentioned interval $2 \%$ deviation of our results of calculations from experimentally measured values (Figure 3). Thus, the traditional analytic theory of averaging properties of the crystals may be used to find a link between the (anisotropic) properties of the individual grains and the effective macroscopic elastic behavior of polycrystalline materials for large volumes of material. A similar conclusion is also confirmed by experimental measurements of barium titanate at different temperatures in [12].

Sheets of alloy ZE10 are anisotropic even in the initial state, as it follows from the results of measurement and calculation of elastic anisotropy. The coefficient of experimental anisotropy of Young's modulus is equal to $2.8 \%$. For calculated anisotropy of Young's modulus this coefficient is $1.6 \%$. We calculated the anisotropy coefficient of Young's modulus $E$ by means of relation

$$
A=\frac{E_{\max }-E_{\min }}{E_{\min }} \cdot 100 \% .
$$



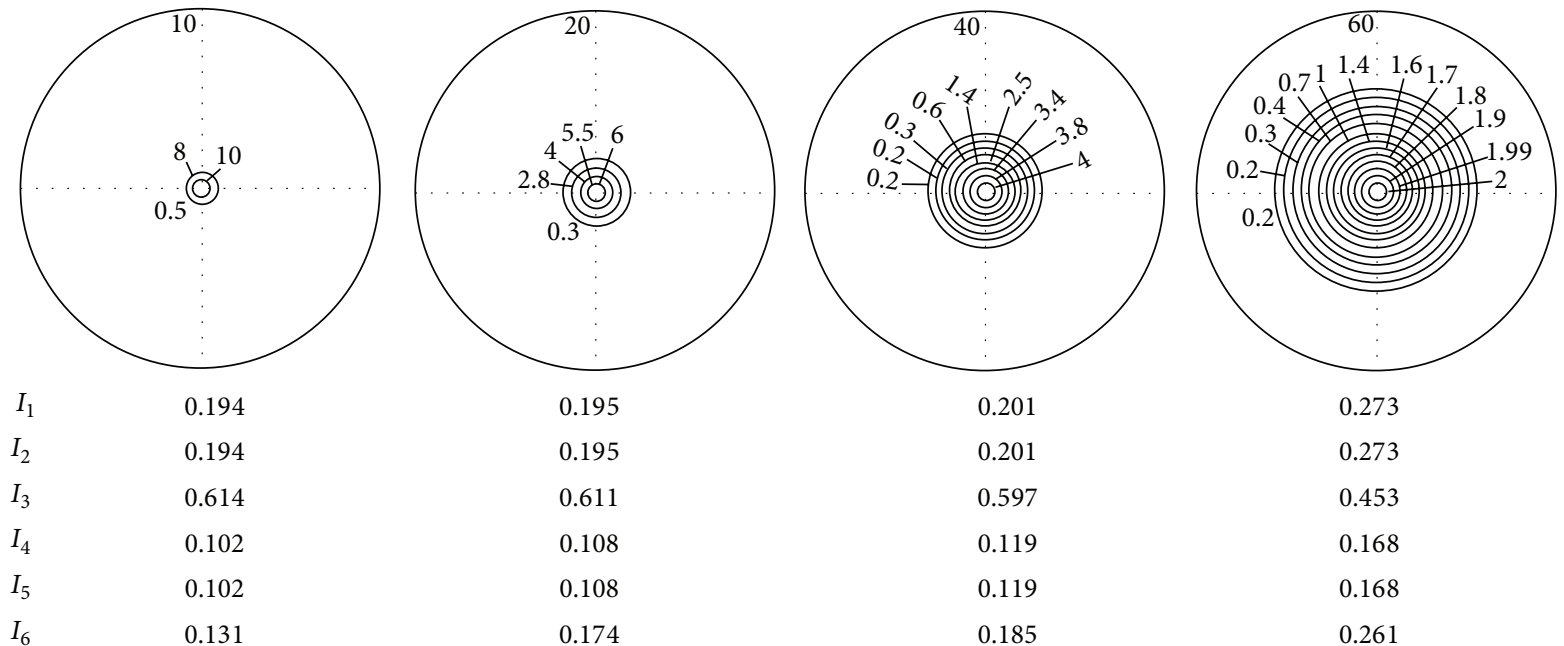

FIGURE 4: Change of ICT with increasing scattering basis poles [0001]. Scattering angle is shown in the upper-portion of each PF.
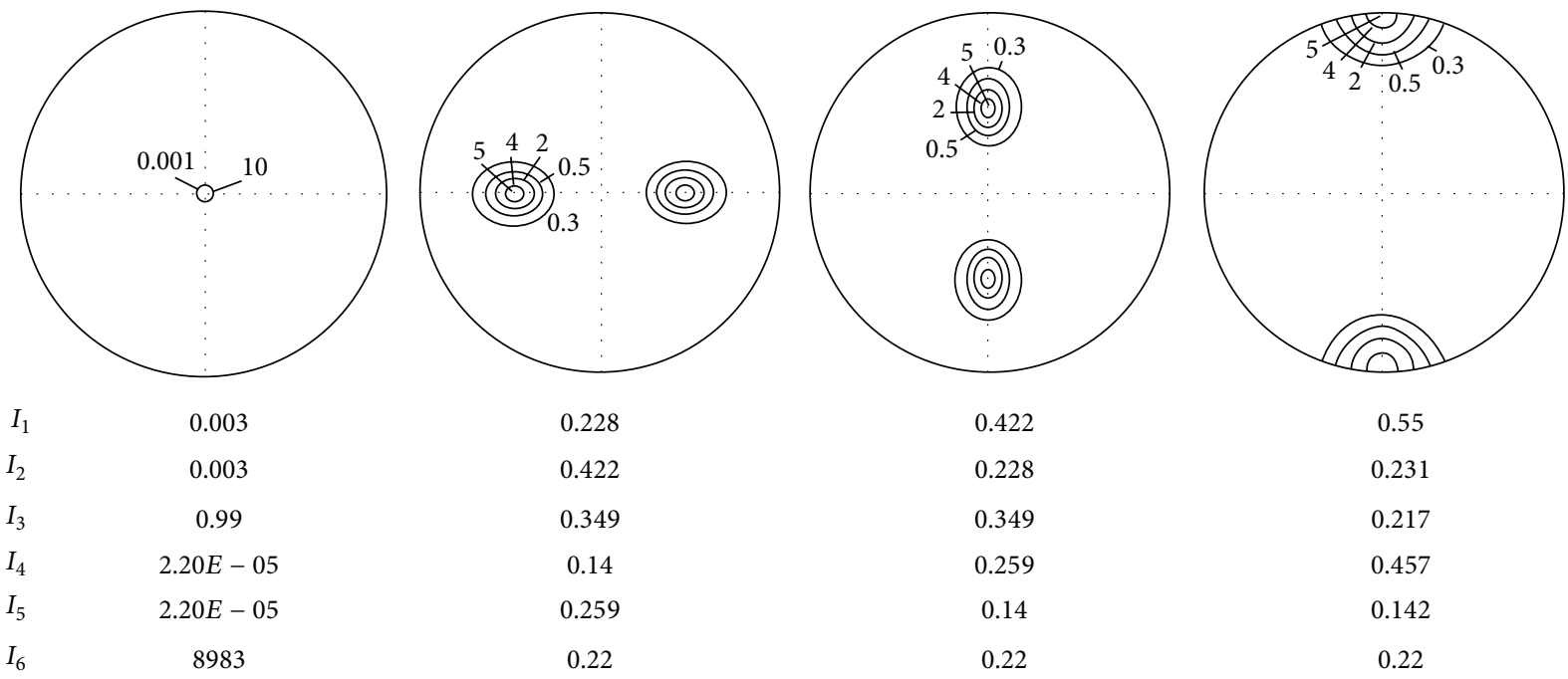

FIgURE 5: Change of ICT with increasing tilt of hexagonal axis [0001] to the plane of sheet.

The maximum of Young's modulus takes place in the RD. The minimal value is observed at the angle $45^{\circ}$ to the RD. On Figure 3 it is visible also that Young's modulus in the RD is greater than in the TD. This is contrasted to the anisotropy of Young's modulus in sheets of magnesium alloy AZ31 [13].

It should be noted that the "effect of texture" is rarely taken into account in engineering designing due to the complexity of description of textures by conventional methods. Method of the representation of texture in terms of the ODF is complicated and requires special training of engineers. Method of ideal orientations does not allow directly estimating the anisotropy of properties caused by the texture. The approach described here, which is based on the set of the integral characteristics of texture, uses a minimal number of parameters that is sufficient to predict the properties in various directions of a sheet or product. These characteristics may be used for certification of the texture state of sheet metal. For example, by modeling of ICT for different types of textures it may be seen [8] that the simultaneous growth of the ICT corresponds to an increase of scattering of poles [0001] by angles of azimuth and meridian (Figure 4).

The increasing of the difference between these characteristics is responsible for development of additional peaks removed from the center of the PF. For example, if $I_{2}>I_{1}$, and $I_{4}>I_{5}$, the texture is corresponded to the deviation from the center of the PF to RD, and vice versa, if the $I_{2}<I_{1}$ and $I_{4}<I_{5}$, then the texture is corresponded to the deviation from the center of the PF to TD (Figure 5). Naturally, there are possible more complicated cases (Figure 1, Table 1).

\section{Conclusion}

(1) Texture of sheet of magnesium alloy ZE10 (1.3\% Zn, $0.15 \% \mathrm{Zr}$, and $0.2 \%$ of REM) after industrial processing is characterized by two pole density maxima, spaced from the center of the pole figure $\{0002\}$ in the 
$\mathrm{RD}$ and TD on angles $45^{\circ}$. The corresponding integral characteristics of texture $\left(I_{i}\right)$ make up $I_{1}=0.267466$; $I_{2}=0.327686 ; I_{3}=0.404848 ; I_{4}=0.150986 ; I_{5}=$ $0.191116 ; I_{6}=0.04711$.

(2) Integral characteristics of texture include the necessary information to predict tensor properties in any direction and may be recommended for certification of the sheet materials on the textural characteristics

(3) Using the results of the Fourier analysis of the experimental anisotropy of Young's modulus of alloy sheet ZE10 and the integral characteristics of the texture, obtained by means of X-ray analysis, the value of the $s_{11}$ and the certain combinations of the constants compliances of the single crystal of alloy ZE10 were determined, which amounted to $s_{11}=2.29$. $10^{-11} \mathrm{~Pa}^{-1} ; a=s_{13}-s_{11}+(1 / 2) s_{44}=-0.1 \cdot 10^{-11} \mathrm{~Pa}^{-1}$; $b=s_{11}+s_{33}-2 s_{13}-s_{44}=0.128 \cdot 10^{-11} \mathrm{~Pa}^{-1}$. Calculated and measured curves of the anisotropy of Young's modulus in the plane of the sheet alloy ZE10 differ by no more than $2 \%$.

\section{Conflict of Interests}

The authors declare that there is no conflict of interests regarding the publication of this paper.

\section{References}

[1] Y. Chino, K. Sassa, and M. Mabuchi, "Texture and stretch formability of Mg-1.5 mass\%Zn-0.2 mass\%Ce alloy rolled at different rolling temperatures," Materials Transactions, vol. 49, no. 12, pp. 2916-2918, 2008.

[2] "Magnesium-based alloy for wrought applications," http://www .sumobrain.com/patents/wipo/Magnesium-based-alloy-wrought-applications/WO2011146970A1.html.

[3] "Elastic moduli: overview and characterization methods. Technical Review ITC-ME/ATCP," http://www.atcp-ndt.com/ images/products/sonelastic/articles/RT03-ATCP.pdf.

[4] H.-J. Bunge, Mathematische Methoden der Teksturanalyse, Akademie, Berlin, Germany, 1969.

[5] A. A. Bryukhanov and A. R. Gokhman, "Integral characteristics of the texture of cubic and hexagonal metals," Izvestiâ vysših učebnyh zavedenij. Fizika, no. 9, pp. 127-131, 1985 (Russian).

[6] A. A. Bryukhanov and A. R. Gokhman, "Calculation method for determining of texture parameters of the tensor properties of cubic and hexagonal metals," Zavodskaya Laboratoriya, vol. 53, no. 3, pp. 572-578, 1987 (Russian).

[7] F. Koutný, "Elementary numerical methods \& Fourier analysis," http://www.koutny-math.com/.

[8] A. A. Bryukhanov, N. A. Volchok, and T. S. Sovkova, "Effect of cold rolling on the characteristics of texture and anisotropy properties of alpha-alloy Ti-3Al-1,5V," Materialy, no. 4, pp. 9-14, 2010 (Russian).

[9] G. Wassermann and J. Greven, "Texturen metallischer Werkstoffe," in Zweite Neubearbeitet und Erweiterte Auflage, Springer, Berlin, Germany, 1962.

[10] H. P. R. Frederikse, "Elastic constants of single crystals," http:// www.docstoc.com/docs/45454109/Elastic-Constants-of-Single-Crystals.
[11] M. Kamaya, "A procedure for estimating Young's modulus of textured polycrystalline materials," International Journal of Solids and Structures, vol. 46, no. 13, pp. 2642-2649, 2009.

[12] J. M. J. den Toonder, J. A. W. dan Dommelen, and F. P. T. Baaijens, "The relation between single crystal elasticity and the effective elastic behaviour of polycrystalline materials: theory, measurement and computation," Modelling and Simulation in Materials Science and Engineering, vol. 7, no. 6, pp. 909-928, 1999.

[13] A. A. Bryukhanov, Y. Zil'berg, M. Schaper et al., "Effect of cold straightening the texture and anisotropy properties of magnesium alloy AZ31 sheets," Deformatsiya i Razrushenie Materialov, no. 8, pp. 34-41, 2010 (Russian). 

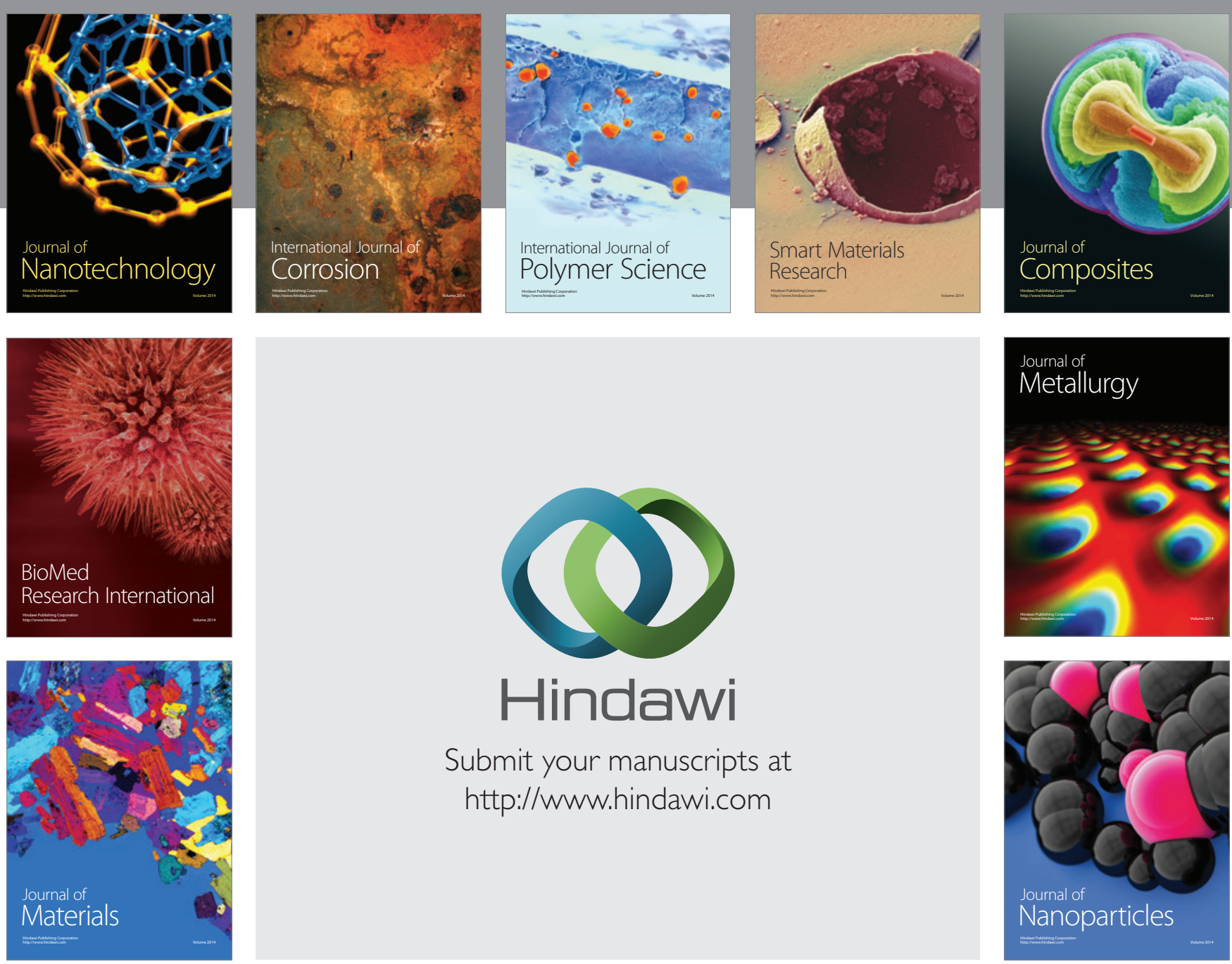

Submit your manuscripts at http://www.hindawi.com
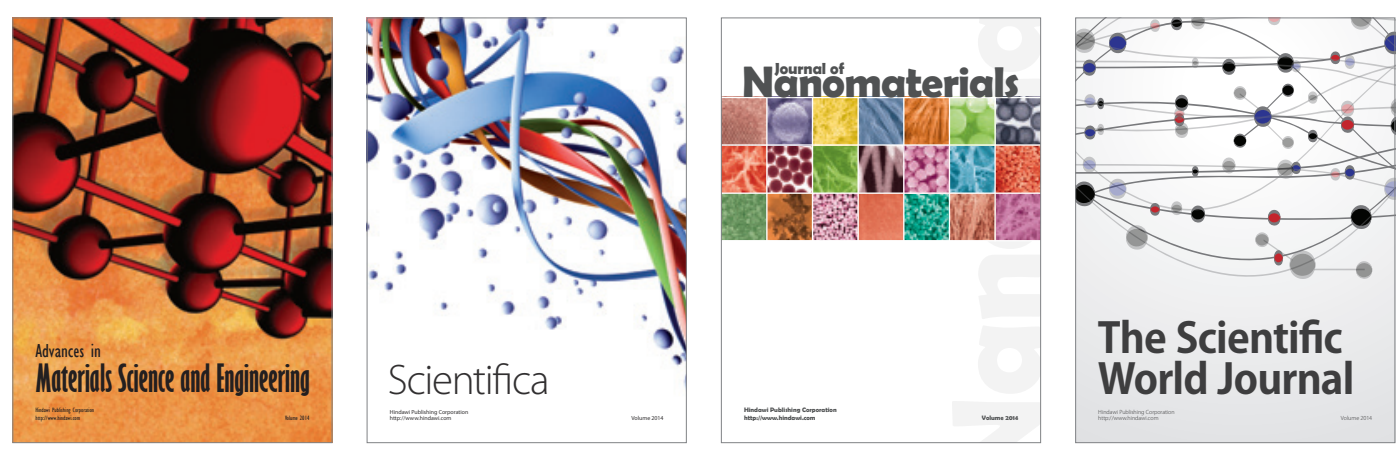

\section{The Scientific World Journal}
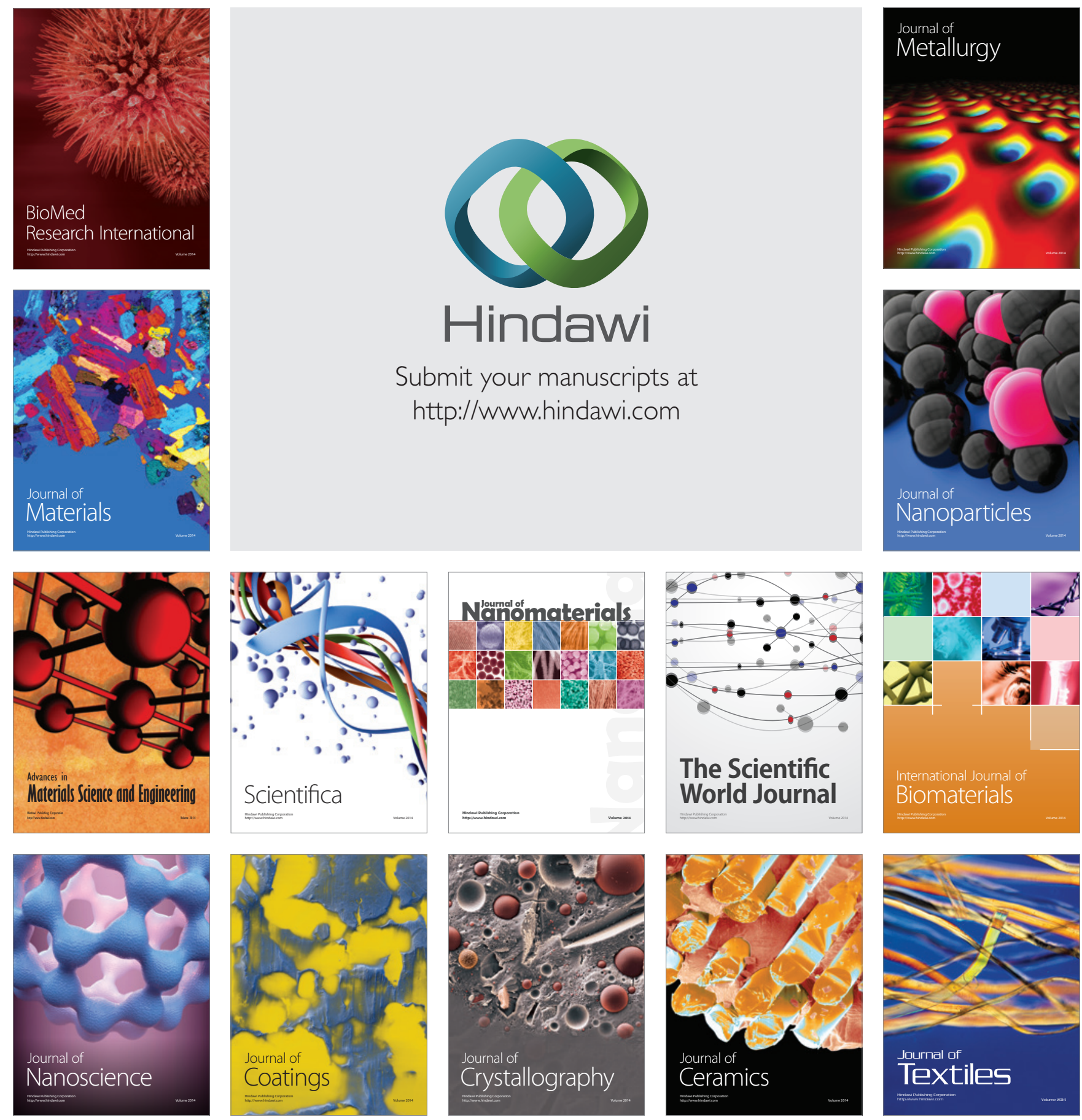